\title{
Correction to: Moyennes effectives de fonctions multiplicatives complexes
}

\section{Gérald Tenenbaum ${ }^{1}$ (iD}

Published online: 15 July 2020

(c) Springer Science+Business Media, LLC, part of Springer Nature 2020

\section{Correction to: Ramanujan J (2017) 44:641-701 https://doi.org/10.1007/s11139-017-9949-7}

The original version of the article unfortunately contained a number of inaccuracies, which even though did not invalidate the statements, may perturb the reader. We present here a list of necessary corrections. Equation and statement numbers refer to [1].

(i) Theorem 1.1 and Corollary 2.1 are valid uniformly when $f$ and $r$ belong to the slightly smaller class of multiplicative functions for which the second condition (1.9) is strengthened to

$$
\sum_{\substack{p^{v} \leqslant x \\ \nu \geqslant 2}} \frac{\left|f\left(p^{\nu}\right)\right|\left(\log p^{\nu}\right)^{2}}{p^{v}} \leqslant B .
$$

(ii) A term $\mathrm{e}^{Z(x ; r)} / \log x$ should be added inside the curly brackets of Eq. (1.12) and a term $1 / \log x$ should be added inside those of Eq. (2.3). Alternatively, one may leave the quantities inside brackets unchanged but restrict in both statements the range for $T$ to $\left[1,(\log x)^{2}\right]$.

(iii) Theorem 1.2 is actually valid for $\left.\left.\delta_{1} \in\right] 0, \frac{2}{3} \mathfrak{b}\right]$. No change in the proof is necessary.

(iv) Equation (3.2) is the usual form in which Gallagher's bound (3.1) is employed. Contrary to what is stated in the remark following the statement of Lemma 3.1, this form is not generically weaker, although this is indeed often the case, in particular if $\delta_{m} \gg \delta_{n}$ whenever $\left|\lambda_{m}-\lambda_{n}\right| \leqslant 1 / T$.

(v) For the reduction of the proof of Theorem 1.1 to the case of exponential multiplicativity, an appeal to (1) yields, by a similar argument to that displayed on p. 657 , that

The original article can be found online at https://doi.org/10.1007/s11139-017-9949-7.

Gérald Tenenbaum

gerald.tenenbaum@univ-lorraine.fr

1 Institut Élie Cartan, Université de Lorraine, BP 70239, 54506 Vandœuvre-lès-Nancy Cedex, France 


$$
Q(y):=\sum_{n>y} \frac{|h(n)|}{n} \ll \frac{1}{(\log y)^{2}} \quad(y \geqslant 2) .
$$

Inserting an extra term $\mathrm{e}^{Z(x ; r)} / \log (2 x / n)$ inside curly brackets of (3.10) and taking account of (2) yields the required estimate.

\section{Reference}

1. Tenenbaum, G.: Valeurs moyennes effectives de fonctions multiplicatives complexes. Ramanujan J. 44(3), 641-701 (2017)

Publisher's Note Springer Nature remains neutral with regard to jurisdictional claims in published maps and institutional affiliations. 